\title{
Anabases
}

\section{Les sociologues parmi les antiquisants : les contributions d'Henri Hubert et de Paul Huvelin au Dictionnaire des Antiquités Grecques et Romaines}

\section{Rafael Faraco Benthien}

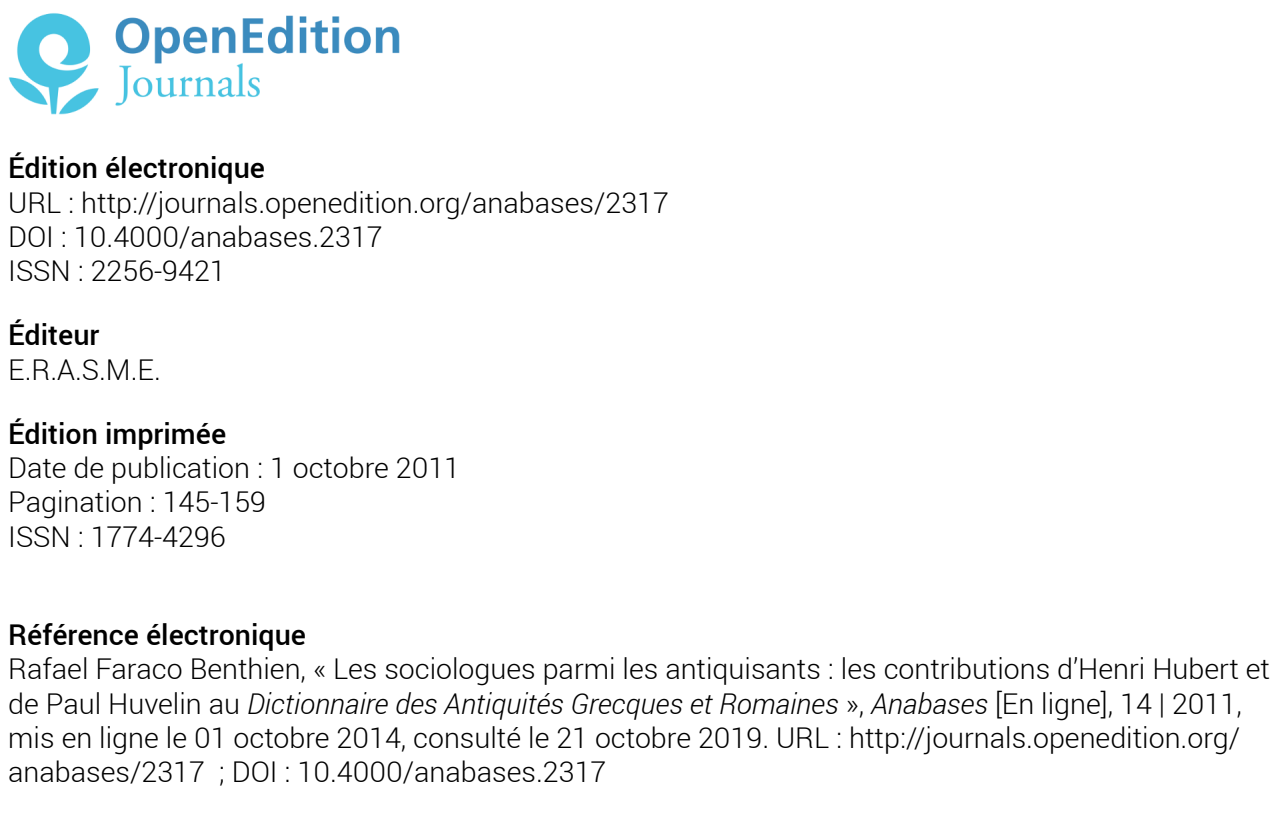

(c) Anabases 
Anabases 14 (2011), p. 145-159.

\title{
Les sociologues parmi les antiquisants : les contributions d'Henri Hubert et de Paul Huvelin au Dictionnaire des Antiquités Grecques et Romaines ${ }^{1}$
}

Rafael Faraco Benthien

\begin{abstract}
Au COURS DES QUARANTE DERNIÈRES ANNÉES, période marquée par un intérêt renouvelé et croissant pour l'histoire de l'école dite durkheimienne, beaucoup de choses ont été dites sur les rapports entretenus entre celle-ci et d'autres groupes actifs au sein de l'Université française, en particulier les philosophes, les historiens et les linguistes. Il s'avère que ces importants travaux sont loin d'avoir épuisé la richesse d'un sujet qui, pour cette raison, requiert non seulement d'autres recherches ponctuelles, mais aussi une mise en parallèle des résultats obtenus jusqu'à aujourd'hui. En ce sens, une option intéressante, et encore peu explorée, serait de présenter un tableau mettant en relief les liens unissant
\end{abstract}

1 Le présent article découle de mes travaux de recherche dans le cadre du doctorat que je réalise à l'université de São Paulo sous la direction de Francisco Murari Pires, en tant que boursier de la Fundação à Pesquisa do Estado de São Paulo (FAPESP). Ils sont centrés sur les rapports entre sociologues et antiquisants pendant la III ${ }^{e}$ République française. Je profite de l'occasion pour remercier Christine Lorre et Pascal Payen, dont les indications bibliographiques m’ont été précieuses. J'aimerais également témoigner ma reconnaissance aux organisateurs de la journée d'études " Henri Hubert et la Mythologie Républicaine », qui a eu lieu à l'université de Chicago à Paris, le 11 juin 2010. Une première version de ce texte y a été présentée. Je ne peux pas oublier que Anaïs Boucher a bien voulu se charger de la correction de cet article. Qu'elle en soit aussi remerciée. En ce qui concerne le Dictionnaire des Antiquités Grecques et Romaines, nous avons utilisé ici l'édition mise en ligne par l'université de Toulouse (http ://DAGR.univ-tlse2.fr/sdx/DAGR/index.xsp). 
les sociologues, les hellénistes et les latinistes. Combler une telle lacune nous semble présenter un caractère d'autant plus urgent que l'espace occupé par la Grèce et la Rome antiques dans l'organisation du système d'enseignement français jusqu'à la première moitié du Xxe siècle était central ${ }^{2}$. La langue et la littérature de ces deux civilisations constituaient en effet la base d'une éducation scolaire largement diffusée et valorisée. Elles offraient par ailleurs aux sociologues des champs de recherche et d'activité professionnelle particulièrement féconds. N'oublions pas que des personnalités comme Henri Hubert, Antoine Meillet, Paul Huvelin, Pierre Roussel, Henri Jeanmaire et Louis Gernet consacrèrent leur carrière, du moins une part de celle-ci, aux études gréco-latines. Cette liste pourrait même être sensiblement élargie si nous prenons en compte tous ceux qui se prévalurent des Anciens à des fins ponctuelles de comparaison.

C'est dans cette voie que nous avons choisi d'inscrire notre étude. Nous présenterons donc une analyse de textes qui furent publiés par des chercheurs appartenant au cercle de Durkheim dans cet ouvrage qui fut la plus grande encyclopédie illustrée de l'Antiquité classique jamais conçue en France, à savoir le Dictionnaire des Antiquités Grecques et Romaines $(D A G R)$. Le découpage tel qu'il a été conçu pour les objectifs de notre recherche se justifie non seulement en raison de l'importance historique de ladite publication, sur laquelle nous reviendrons ci-après, mais aussi parce que quatre des cinq volumes du dictionnaire qui furent publiés à partir de 1898, soit après le lancement de L'Année sociologique, comprennent plusieurs articles signés par les sociologues durkheimiens. Certaines questions se posent dès lors à nous. Comment expliquer la présence constante de ces sociologues et la logique de leur recrutement ? Quels thèmes abordèrent-ils ? De quelle manière l'approche sociologique apparaît-elle dans le développement de ces thèmes ? Et pour terminer, par-delà la contribution des sociologues, quel intérêt le travail de l'école durkheimienne suscita-t-il dans l'ensemble de l'ouvrage ? Mais avant de répondre à ces questions, il convient de présenter dans les grandes lignes ce que fut exactement le $D A G R$.

\section{Le Dictionnaire des Antiquités Grecques et Romaines}

Le Dictionnaire des Antiquités Grecques et Romaines, édité par Hachette, parut de façon ininterrompue, mais à un rythme irrégulier, de 1877 à 1919. Ainsi, entre la publication du premier volume, relatif aux lettres A-B, et celle du deuxième volume, centré exclusivement sur la lettre $\mathrm{C}$, nous observons que dix ans se sont écoulés. En revanche, le troisième volume, consacré aux lettres D-E, parut en 1892, soit cinq ans à peine après le précédent. Par la suite, nous remarquons une tendance à la stabilité, avec des inter-

2 Au-delà de l'analyse de Durkheim lui-même, qui fait figure de pionnier (cf. É. DuRKHEIM, L'évolution pédagogique en France, Paris, 1999, p. 351-382), voir F. Ringer, Fields of Knowledge, Cambridge-Paris, 1992, p. 40-45. 
valles de trois à quatre ans entre deux publications, avec uniquement une interruption lors de l'éclatement de la Guerre 14-18, comme l'indique le tableau 1. Il faut ajouter qu'anticipant sur la sortie des grands tomes, la maison d'édition offrait périodiquement au public de petits fascicules, qui contribuèrent beaucoup à dynamiser la production et la circulation de l'ouvrage.

\begin{tabular}{|c|c|c|c|}
\hline Tome & Année & Nombre de notices & Nombre de pages \\
\hline A-B & 1877 & 665 & 756 \\
\hline C & 1887 & 499 & 947 \\
\hline D-E & 1892 & 402 & 946 \\
\hline F-G & 1896 & 193 & 769 \\
\hline H-K & 1900 & 269 & 880 \\
\hline L-M & 1904 & 387 & 1262 \\
\hline N-Q & 1907 & 483 & 808 \\
\hline R-S & 1911 & 403 & 792 \\
\hline T-Z & 1919 & 420 & 1077 \\
\hline
\end{tabular}

Tableau $1-D A G R$ : périodicité et contenu

S'il est vrai que l'année du début de la publication du DAGR correspond à la première décennie d'une III ${ }^{\mathrm{e}}$ République encore fragile, il nous faut reculer un peu plus dans le temps pour arriver à nous faire une idée de ses origines réelles. C'est en 1855 que la maison d'édition Hachette et Charles Daremberg - l'inventeur du projet signèrent le contrat établissant le contenu de l'ouvrage et le public ciblé. En dépit des changements apportés par la suite, il y était établi que les articles devraient traiter de divers aspects des civilisations grecque et latine, avec une insistance tant sur les coutumes, les croyances et les lois que sur la vie matérielle et sur les monuments conservés ${ }^{3}$. Quant à ce qui touchait le public ciblé, l'objectif était de rendre l'érudition accessible au profane. D'où le souci de doter les articles d'illustrations et, quand le cas s'y prêtait, d'une bibliographie générale. Les spécialistes ne furent pas oubliés pour autant : de copieuses notes en bas de page leur seraient destinées et, en plus de présenter en détail l'état de l'art sur une question déterminée, elles permettraient de justifier des critiques ou d'avancer des thèses.

Ainsi, de 1855 à 1919, c'est-à-dire entre la signature du contrat et la conclusion de l'ouvrage, soixante-quatre ans s'écoulèrent. Une telle longévité permet d'entrevoir, sous le vernis de la continuité, une des périodes les plus riches de l'histoire intellectuelle française. Voilà peut-être le plus grand intérêt du DAGR pour le chercheur d'aujourd'hui : sous l'angle des transformations qui touchèrent la direction et le recrutement des

3 Au départ, le DAGR devait aussi traiter de l'Antiquité Orientale et du Moyen Âge. Sur ces changements, voir C. VAlENTI. " "Daremberg et Saglio" ou "Saglio et Pottier" ? La difficile gestation d'un dictionnaire savant ", Anabases 4 (2006), p. 159-167. 
collaborateurs, l'ouvrage présente une synthèse du passage d'un modus vivendi centré sur les salons fréquentés par les érudits et sur les académies locales ou nationales vers un registre proprement et principalement universitaire. Penchons-nous maintenant, comme sur un premier indice de ce processus, sur la trajectoire de son fondateur et premier directeur, Charles Daremberg.

Né dans la ville de Dijon en 1817, après avoir été élève dans un séminaire de province, Daremberg suivit la carrière de médecin. Mais il ne fut pas un quelconque médecin : d'abord et avant tout, il fut un érudit. Sous l'infuence du cercle positiviste rassemblé autour d'Émile Littré, Daremberg finit ainsi par consacrer la majeure partie de son temps à l'étude de l'histoire de la médecine. Son premier grand travail dans ce domaine fut une thèse qu'il soutint en 1841 à la faculté de médecine de Paris, dans laquelle il étudiait l'œuvre de Galien. Après une période où il exerça la fonction de bibliothécaire dans cette faculté, il se lia à l'Académie des inscriptions, qui l'envoya à Rome pour cataloguer des manuscrits relatifs à la médecine antique et conservés au Vatican. Selon sa biographe Danielle Gourevitch, cette expérience au milieu des archives fut déterminante pour Daremberg, au point de faire germer en lui l'idée de publier un dictionnaire ${ }^{4}$. De fait, peu d'années après avoir quitté l'Italie, il entama des négociations avec Hachette, qui ne devaient produire de résultats vraiment concrets qu'en 1877 , soit cinq ans après sa mort.

L'autre co-fondateur de l'ouvrage, Edmond Saglio, servit d'intermédiaire entre cet érudit lié aux salons scientifico-positivistes et les universitaires de la III République. Né à Paris en 1828, bachelier et licencié en droit, Saglio côtoyait ces deux mondes. En effet, durant une période considérable de sa vie, il se partagea entre son travail d'avocat au sein du ministère de la Justice et ses études touchant l'histoire de l'art et l'archéologie. Au fil des ans, sous le parrainage du spécialiste en études médiévales Jules Quicherat, dont il avait suivi les cours à l'École des Chartes, Saglio prend ses distances avec le droit. En 1879, il vint ainsi à occuper la charge de conservateur adjoint au musée du Louvre, qu'il laissa plus tard, en 1893, pour assurer la direction du musée de Cluny.

Il convient de souligner que l'archéologie, un des principaux centres d'intérêt de Saglio, représentait l'avant-garde du mouvement de professionnalisation des différents corps lettrés français ${ }^{5}$. Sous l'impulsion du succès politico-scientifique des fouilles sur le territoire et en dehors de la France, ce fait se traduisit par la création de nouvelles institutions essentiellement tournées vers la recherche, comme le Musée des antiquités nationales et l'École pratique des hautes études, et aussi par la réforme des institutions anciennes. Préalablement timorées, les reformulations du système d'enseignement

4 D. Gourevitch, "Daremberg, Charles ", Dictionnaire Critique des Historiens de l'Art, Édition en lignes de l'INHA, http ://www.inha.fr/spip.php ?article2265 (site consulté le 20 mai 2010).

5 È. Gran-Aymerich, Naissance de l'archéologie moderne. 1798-1945, Paris, CNRS, 1998, p. 41-202. 
français s'accélérèrent et s'approfondirent après la défaite militaire contre la Prusse et grâce à la consolidation de la III ${ }^{e}$ République. Recruté par Daremberg vers le milieu des années 1860, Saglio accompagna de près ces changements et fut sans doute le grand responsable de la sortie du premier volume du $D A G R$. Mais comme la logistique d'une œuvre aussi monumentale s'avérait fort complexe pour un seul et unique homme, un jeune helléniste, entièrement formé dans le contexte des cadres universitaires nouveaux, fut invité à se joindre au projet. Il s'agissait d'Edmond Pottier.

Si l'université moderne, institution où doit prédominer l'éducation visant la production d'une connaissance scientifique originale, se consolida en France aux alentours de 1880, comme le soutiennent les spécialistes de l'histoire du système d'enseignement français, il faut considérer qu'Edmond Pottier appartenait à la première génération de ce renouveau ${ }^{6}$. Né à Sarrebrück en 1855, il passa par le lycée Condorcet, pour rejoindre ensuite l'École normale supérieure (ENS). Membre de la promotion de 1874 et premier à l'Agrégation de Lettres en 1877, Pottier se fit remarquer plus tard pendant ses années de travail à l'École française d'Athènes, où il eut l'occasion de s'instruire sur ce qu'il y avait de plus moderne en archéologie, en épigraphie et en histoire antique. Son retour en France coïncida avec le début de sa carrière comme professeur à l'université. Sa charge débuta aux facultés des lettres de Rennes et de Toulouse, entre 1880 et 1883, avant de se poursuivre à Paris. Là, il donna des cours d'archéologie à l'École des beaux-arts et aussi à l'École du Louvre, assumant un poste de conservateur du Louvre, d'abord comme attaché libre (1884), puis comme adjoint (1897). Pottier devint la référence française en céramiques grecques et occupa aussi la fonction de co-directeur dans une des revues scientifiques françaises les plus anciennes et les plus prestigieuses : la Revue Archéologique.

De Daremberg à Pottier, ce sont grosso modo trois générations réunies autour de la direction d'un même projet éditorial. Mais chacune de ces générations présente par rapport aux autres des modalités distinctes de capital social et intellectuel. Daremberg ne devait pas consacrer l'essentiel de sa vie à la recherche et à l'enseignement universitaires. Le positivisme, qui était sa théorie de référence, se développait en dehors de l'université, dont il atteignit seulement les marges au cours de la deuxième moitié du $\mathrm{XIX}^{\mathrm{e}}$ siècle ${ }^{7}$. Quant à Saglio, bien qu'ayant débuté sa carrière comme érudit mondain, il adhéra aux premiers mouvements d'avant-garde de la recherche universitaire conçue selon de nouveaux moules. Il fréquenta l'École des Chartes et se joignit aux équipes des musées nationaux. Pottier, pour sa part, se différencia de ses prédécesseurs par une

6 En plus des auteurs cités dans la note 1, nous renvoyons à la synthèse proposée par F. Mayeur, Histoire de l'enseignement et de l'éducation. III. 1789-1930, Paris, 2004, p. 581-635. Quant aux informations sur la trajectoire de Pottier, voire R. LANTIER et C. Picard, "Edmond Pottier ", Revue Archéologique 2 (1934), p. v-xxviii.

7 J. Heilbron, Naissance de la sociologie, Marseille, 2006, p. 269-368. 
carrière entièrement universitaire. Il la commença à l'ENS, la compléta lors de stages à l'étranger, et la conclut par des activités de professeur-chercheur.

Partant des données quantitatives présentées par Andurand, Cazenave et Delmas sur la morphologie du $D A G R$, nous constatons que le profil incarné par Pottier s'imposa au fil des ans à la majorité des collaborateurs de l'ouvrage ${ }^{8}$. Finalement et sans avoir commencé de cette façon, le DAGR devint une sorte d'extension de l'université de la III République, car il incorporait les transformations survenues dans l'univers intellectuel français. Voilà d'ailleurs un argument clé pour comprendre la présence des sociologues proches de Durkheim. À l'inverse des autres groupes qui leur disputaient le contrôle des sciences sociales à la fin du XIX ${ }^{\mathrm{e}}$ siècle, les membres de l'équipe de L'Année sociologique avaient eux aussi un profil universitaire? . Nous allons voir lesquels de ces membres adhérèrent au $D A G R$, et les raisons qui les conduisirent à agir de cette façon.

\section{Henri Hubert : un jeune homme prometteur parmi les antiquisants}

En termes de chronologie, les premières contributions apportées au DAGR par un sociologue proche de Durkheim sont attribuables à Henri Hubert. Il rédigea deux notices en tout, une pour le cinquième volume et l'autre pour le sixième, comme nous le voyons dans le cadre ci-dessous.

\begin{tabular}{|l|c|c|c|c|}
\hline Notices & Volume & $\begin{array}{c}\text { Année } \\
\text { de publication }\end{array}$ & $\begin{array}{c}\text { Nombre de } \\
\text { colonnes }\end{array}$ & Pagination \\
\hline KYRÈNÉ & H-K & 1900 & 5 & $873-876$ \\
\hline MAGIA & L-M & 1904 & 51 & $1494-1521$ \\
\hline
\end{tabular}

Tableau 2 - Henri Hubert et le DAGR

8 A. Andurand, J. Cazenave, S. Delmas, «Appréhender le Dictionnaire des Antiquités grecques et romaines par sa table d'auteurs. Les données statistiques comme outil d'analyse ", Anabases 4 (2006), p. 219-223.

9 Sur les bases d'appui institutionnelles des durkheimiens et des autres groupes qui coexistaient et s'affrontaient par rapport à la destinée des sciences sociales, voir : T.N. CLARK, Prophets and Patrons, Cambridge, 1973 ; V. KARADY, " Durkheim, les sciences sociales et l'Université : bilan d'un semi-échec ", Revue française de sociologie 17 (1976), p. 267-311; V. Karady, "Stratégies de réussite et modes de faire-valoir de la sociologie chez les durkheimiens ", Revue française de sociologie 20 (1979), p. 49-82 ; L. MucCHIELli, La découverte du social, Paris, 1998 ; M. Consolim, "Gabriel Tarde e as ciências sociais francesas ", Mana 14/1 (2008), p. 269-298. 
Avant de parler du contenu relatif à ces contributions, il importe de situer celles-ci dans la trajectoire d'Henri Hubert ${ }^{10}$. Âgé seulement de 28 ans en 1900, ce n'est certainement pas en tant que chercheur prestigieux et achevé qu'il débuta dans le projet du $D A G R$. Il n'avait publié aucun livre. Il ne pouvait se prévaloir que de quelques articles, parmi lesquels se détachaient une étude sur l'État papal et un essai sur le sacrifice écrit en collaboration avec Marcel Mauss, deux travaux datant de $1899^{11}$. Quant à sa position institutionnelle, outre la charge non rémunérée d'attaché libre exercée au sein du Musée des antiquités nationales depuis 1898, il devrait attendre 1901 pour que lui soit attribuée celle de maitre de conférences à l'École pratique des hautes études (EPHE). En résumé, le Hubert qui travailla pour le $D A G R$ n'était autre qu'un débutant dans le monde universitaire. Néanmoins, en tout cas aux yeux des directeurs de la publication, il semble qu'il soit passé pour un jeune homme responsable, et surtout prometteur.

Ses rapports avec Salomon Reinach, son directeur au Musée des antiquités nationales, ont sans aucun doute fait valoir son nom dans les cercles des antiquisants, sans qu'il soit toutefois possible de déduire de ce fait la participation de Hubert au DAGR. Une telle déduction demanderait de clarifier préalablement comment la confiance de Reinach lui fut acquise, et de confirmer par ailleurs l'influence réelle de celui-ci sur les opinions de Saglio et de Pottier. Pour échapper à ce qui ne serait qu'une simple chaîne de causes et d'effets, il importe donc, comme le suggérait Max Weber, d'envisager les conditions sociales de production et de reconnaissance d'une vocation. Le fait est qu'Hubert, peut-être plus que n'importe quel autre membre de l'équipe originelle de L'Année sociologique, présentait toutes les conditions requises pour être perçu comme un jeune chercheur dont le potentiel dans le champ des études gréco-latines était grand. Sa formation explique ce phénomène, du moins en partie. Détenteur d'un prix de version grecque au Concours général au temps du lycée Louis-le-Grand, Hubert continua d'étudier en profondeur les langues et les textes anciens à l'ENs, d'où il sortit agrégé d'histoire en 1895, et aussi à l'EPHE. De cette époque encore date une certaine expérience en épigraphie, comme en témoigne son premier article intitulé " Deux inscriptions métriques d'Asie Mineure ${ }^{12}$ ".

À cet entraînement scolaire digne d'un antiquisant, le jeune Hubert sut associer les contacts sociaux. L'analyse de sa correspondance passive, conservée dans les archives

10 Pour un aperçu récent et assez complet de la carrière et des publications d'Henri Hubert, voir C. LORRE, "Hubert, Henri ", Dictionnaire Critique des Historiens de l'Art, Édition en lignes de l'INHA, www.inha.fr/article2370 (site consulté le 22 mai 2010).

11 Voir, respectivement, H. Hubert, «Étude sur la formation des États de l'Église : les papes Grégoire II, Grégoire III, Zacharie et Étienne II et leurs relations avec les empereurs iconoclastes (726-757)", Revue historique 64 (1899), p. 1-40, 241-272 ; H. HUBERT et M. Mauss, "Essai sur la nature et la fonction social du sacrifice ", L'Année sociologique 2 (1899), p. 29-138.

12 H. Hubert, "Deux inscriptions métriques d'Asie Mineure ", Revue archéologique 1 (1894), p. 308-314. 
de l'Institut Mémoires de l'Édition Contemporaine (IMEC), fait apparaittre des révélations importantes dans ce sens. Échangées dans le cercle de ses camarades de l'ENS, par exemple, les lettres écrites par Paul Perdrizet et Pierre Jouguet, tous deux antiquisants, révèlent une amitié et un respect intellectuel profond à l'égard d'Hubert. Dans une lettre datée de septembre 1894, Jouguet vient même à demander que ce dernier reconsidère sa décision de ne pas se rendre à l'École française d'Athènes, ce qui prouve au moins que le jeune homme se posa un jour la question ${ }^{13}$. Hubert maintint aussi de bons rapports, peut-être même exceptionnellement bons, avec quelques-uns des représentants les plus illustres du champ des études gréco-latines. Parmi ses correspondants de la fin du XIX ${ }^{\mathrm{e}}$ siècle figurent des noms comme celui du latiniste René Cagnat, professeur au Collège de France, ou celui de l'helléniste Amédée Hauvette, de la Sorbonne ${ }^{14}$. Celui-ci lui envoie même des invitations pour des soirées privées et lui demande son aide pour la réalisation de menus travaux. Quant au précédent, qui l'appelle " cher ami ", il lui confie la tâche de s'occuper des inscriptions épigraphiques de Syrie dans un recueil de sources, travail auquel Hubert renonce toutefois au profit de ses amis ${ }^{15}$. De tels exemples sont nombreux ${ }^{16}$.

Les liens d'Hubert avec les latinistes et les hellénistes se manifestent aussi dans ses contacts maintenus avec la Société pour l'Encouragement des Études Grecques en France. Il fut le premier sociologue à devenir membre de cette institution, en 1898. Ce fait, ignoré de ses biographes, montre combien il se sentait à l'aise pour investir professionnellement et affectivement dans un espace où il connaissait les personnes et dont il dominait les codes. Son engagement même, toujours plus grand, par rapport à la publication de L'Année sociologique ne le conduisit pas à s'écarter des antiquisants. En définitive, la Grèce et la Rome antiques, ainsi que les autres peuples "primitifs " d'Europe furent attribués à Hubert lors du partage des objets d'étude que Mauss et lui fixèrent très tôt ${ }^{17}$. C'est ce dont attestent les notices signées par lui dans le DAGR.

L'invitation faite à Hubert pour qu'il écrive la première de ses contributions au $D A G R$ vint de la plume d'Edmond Pottier. Dans une lettre de 1899, ce dernier charge le jeune sociologue de rédiger un texte court sur la déesse éponyme de Cyrène, la colonie

13 Archives de l'IMEC, Fonds Mauss-Hubert, boîte mas 49.

14 Voir les archives de l'IMEC, Fonds Mauss-Hubert, boîte MAS 47 (Cagnat) et MAS 49 (Hauvette).

15 Hubert est intervenu auprès de Cagnat pour que Pierre Jouguet soit chargé de la tâche, provoquant la réaction suivante de son ami : "La démarche que tu as faite auprès de Cagnat me touche beaucoup ; mais je suis confus de te voir abandonner un titre qui te serait très utile et que tu mérites mieux que moi. » Lettre non datée (elle est probablement de 1898), archives de l'IMEC, Fonds Mauss-Hubert, boîte MAS 49.

16 C'est le cas du matériel se trouvant dans les archives de l'IMEC, Fonds Mauss-Hubert, de Franz Cumont (MAS 47), Albert Grenier (MAS 49) et Théodore Reinach (MAS 51).

17 M. Mauss, "L'œuvre de Mauss par lui-même ", Revue française de sociologie 20 (1979), p. 209-220. 
grecque du Nord de l'Afrique ${ }^{18}$. Dans les deux pages et demie consacrées au sujet, nous sommes frappés par l'érudition et par la rigueur méthodologique de l'auteur. En effet, Hubert n'opère pas de tri parmi les récits mythiques et les monuments figurés relatifs à la déesse ; il présente au contraire une liste complète et décrit tous les indices disponibles, en essayant d'identifier les lignes de force censées caractériser la nature de la divinité, et principalement son culte. Tout en conservant en toile de fond une perspective comparatiste, Hubert découvre en Cyrène une divinité liée à la fécondité végétale et à la chasse, dont le mythe aurait été marqué par une théomachie (le combat contre un lion) et par une hiéromachie (l'union avec Apollon). Des auteurs renommés comme Usener et Roscher sont cités par lui, de même que son propre essai sur le sacrifice, qui permettait de mettre en lumière certains invariants présents dans les rituels associés à la déesse. Hubert ne néglige pas non plus l'histoire du culte de Cyrène, soulevant des hypothèses sur son origine et observant son assimiliation à celui d'Artémis. Il est dès lors possible de considérer ce texte comme pleinement intégré aux préoccupations du jeune sociologue. Il est directement lié aux réflexions plus générales des membres du groupe de L'Année sociologique sur les pratiques magico-religieuses. Il anticipe aussi sur les grandes analyses de divinités que lui-même viendra à réaliser plus tard dans le domaine celtique ${ }^{19}$.

Magia, la deuxième et dernière notice publiée par Hubert dans le DAGR, differe de la précédente par sa longueur - 25 pages et demie - et par son audace. L'objectif visé est de rendre compte, en les systématisant, des pratiques magiques existant dans tout le bassin méditerranéen, en insistant sur l'existence d'un ensemble cohérent formé après l'expansion militaire macédonienne et surtout romaine. Ainsi, tout en évoquant la multiplicité de contextes historiques particuliers, une évidence s'impose dans le traitement des sources littéraires (Homère, Hésiode, Hérodote, Diogène Laërce, Apulée, entre autres) et des matières (peintures sur céramique et offrandes votives en plomb) : les traits systémiques - les invariants - semblent l'avoir intéressé au plus haut point. Ce choix, que l'auteur lui-même reconnaît comme provisoire et non scientifique, présente au moins l'avantage de protéger l'analyse de toute tentation de transformation en une paraphrase incontrôlée des sources antiques. Dans cet esprit, l'auteur organise la notice en trois grands blocs : après une brève introduction (p. 1494-1497), il présente l'histoire de la problématique et les principales sources antiques (p. 1497-1506), pour finalement traiter les procédures rituelles et les pouvoirs du magicien, et aussi pour les situer dans des ensembles cérémoniels plus grands (p. 1506-1514).

Sur les thèses défendues ici par Hubert et sur leur place dans la production de L'Esquisse d'une Théorie de la Magie, texte célèbre qu'il viendra à publier avec Mauss en 1904, il n'est pas possible de dire beaucoup plus que ce qui a été formulé par Marcello

18 Archives de l'imec, Fonds Mauss-Hubert, boîte mas 51.

19 Nous faisons allusion ici aux essais rassemblés dans H. Hubert, Divinités gauloises : Sucellus et Nantosuelta, Epona, dieux de l'autre monde, Mâcon, 1925. 
Carastro $^{20}$. Comme l'a bien montré cet helléniste, la toile de fond de la notice renvoie à un contexte plus large que le contexte gréco-latin. Hubert cite à de nombreuses reprises des spécialistes d'autres peuples et civilisations, ce qui lui permet de polémiquer avec Frazer sur les principes généraux de la magie. Il critique surtout la tendance de l'anthropologue britannique à faire dériver toute opération magique d'une logique de sympathie. À l'inverse, ou plutôt en ajout par rapport à la thèse de Frazer, Hubert s'appuie sur des citations de son propre essai sur le sacrifice pour souligner l'importance de l'opposition sacré/profane comme clé explicative de l'ensemble du phénomène magique. Il s'agit en dernière instance d'une anticipation de ce qui serait produit de meilleur en termes de sociologie religieuse par l'équipe de L'Année sociologique. Telle est l'importance théorique de la notice publiée dans le $D A G R$.

Un autre intérêt de cette notice touche au prestige qu'elle octroya au nom d'Hubert parmi les antiquisants. Les deux lettres que lui adressa Saglio, conservées à l'IMEC, le laissent supposer. Si dans la première de ces lettres, datée du 2 juillet 1901, le directeur du DAGR manifeste sa préoccupation par rapport à l'ampleur que la notice était en train de prendre, dans celle du 28 du même mois, il félicite Hubert : "J'admire le courage et la patience avec lesquels vous vous êtes jeté et vous avez marché, en sachant vous reconnaître, dans ce [domaine ?] des sciences occultes ${ }^{21}$. " Dans la même lettre, Saglio invite aussi le sociologue à rédiger un nouvel article, qui devra s'intituler Maleficium. Celui-ci ne figurera toutefois pas dans le dictionnaire. Publiée dès 1902 dans un petit fascicule anticipant sur la sortie du sixième volume, Magia eut un certain impact, spécialement parmi les hellénistes. Dans la Revue des Études Grecques, nous avons pu localiser quatre citations de cette notice, toutes situées entre 1903 et $1907^{22}$, tandis que dans la Revue des Études Anciennes, nous n'en avons localisé qu'une mention, datant de 1920 , ce qui témoigne au moins de ce que le texte continua de circuler durant un temps considérablement long ${ }^{23}$.

20 M. Carastro, " La magie entre histoire et anthropologie. Relire la contribution d'Henri Hubert au DAGR", Anabases 4 (2006), p. 251-255 ; ID., "La fabrique de la notion moderne de magie. Le comparatisme chez Frazer, Hubert et Mauss ", Revista de História edição especial (2010), p. 231-248.

21 Archives de l'Institut de l'édition contemporaine (IMEC). Fonds Mauss-Hubert, boîte MAS 52.

22 Voir P. PERDRIZET, " p. 42-61 ; P. Perdrizet, "Isopséphie ", Revue des Études Grecques (1904), p. 350-360; P. Girard, "Ajax fils de Télamon, étude de mythologie héroïque ", Revue des Études Grecques (1905), p. 1-75; et enfin W. DeonnA, "Talismans magiques touvés dans l'île de Thasos " (1907), p. 373.

23 P. Roussel, "Médée et le meurtre de ses enfants ", Revue des Études Anciennes (1920), p. $157-171$. 


\section{Paul Huvelin, le spécialiste consacré}

Alors que durant la période où il apporta sa contribution au DAGR Henri Hubert n'était qu'un jeune homme aux talents prometteurs, Huvelin occupait quant à lui la position de spécialiste reconnu. Né en 1873, soit un an après Hubert, il acquit des titres et consolida une œuvre personnelle plus tôt que son collègue de L'Année sociologique. En 1904, lorsque son premier texte fut publié dans le $D A G R$, il était déjà docteur en droit et professeur de droit romain à l'université de Lyon. Son titre lui fut conféré en 1897 grâce à la soutenance d'une thèse intitulée l'Essai sur le droit des marchés et des foires, dont le souffle comparatiste et historique embrassait de longues périodes de l'histoire économique dite occidentale, notamment celle de l'Antiquité gréco-romaine. Concernant son poste, il faut dire qu'Huvelin était déjà passé par l'université d'Aix, où il était arrivé vers le milieu de l'année 1898 pour y exercer la fonction de chargé de cours complémentaire d'histoire du droit français. Il s'était rendu à Lyon l'année suivante, à la suite de son succès à l'examen d'agrégation. Sa nomination avait eu lieu un peu plus tard, en 1901, comme professeur d'histoire du droit romain, également à Lyon.

La rapide ascension d'Huvelin s'explique en grande partie par l'inclination de ses investigations intellectuelles. Comme l'a bien montré Frédéric Audren, il inaugura les discussions sur une branche du savoir jusqu'alors pratiquement inconnue en France, à savoir l'histoire du droit commercial ${ }^{24}$. Dans ce cas spécifique, Huvelin rompit avec une vision strictement normative de la loi. Cette vision, en radicalisant l'autonomie du droit, passait sous silence la spécificité des rapports entre la loi et le monde, et plus encore la capacité de la loi d'intervenir dans le monde. Il est bien vrai que la perspective d'Huvelin n'impliquait pas une négation du statut même du droit, ou pour être plus précis, de sa relative indépendance. Mais Huvelin la complexifiait de manière considérable, comme on pourra s'en rendre compte ci-après. Quant à ses études touchant le droit romain, elles opéraient sur des terrains très proches de ceux de la sociologie de la religion produite dans L'Année sociologique. Grosso modo, il cherchait à comprendre comment, à partir d'une source magique ou religieuse, un droit pouvait voir le jour sans être ni de la magie ni de la religion.

Le début de la participation d'Huvelin au DAGR coïncide pratiquement avec son adhésion au projet scientifique dirigé par Durkheim. Grâce aux lettres d'Huvelin adressées à Mauss, et qui sont conservées à l'IMEC, nous savons qu'il se rapprochait déjà du champ de la sociologie universitaire depuis $1899^{25}$. La réciproque était également vraie, étant donné que la production d'Huvelin sur l'histoire de l'ancien droit romain

24 F. Audren, "Paul Huvelin (1873-1923) : juriste et durkheimien ", Revue d'histoire des sciences humaines 4/1 (2001), p. 117-130.

25 Archives de l'imec. Fonds Mauss-Hubert, boîte mas 6.40. La transcription de ces lettres est disponible dans l'article cité de Frédéric Audren (cf. note 24). 
fut bien accueillie parmi les disciples de Durkheim ${ }^{26}$. Nous observons qu'il vint à figurer parmi les collaborateurs réguliers de L'Année sociologique à partir de 1903. Dans le $D A G R$, auquel il participa dès 1904, nous rencontrons en tout dix notices signées par lui, dont la longueur et les thèmes sont variables, comme le montre le tableau ci-dessous.

\begin{tabular}{|c|c|c|c|c|}
\hline Notices & Volume & $\begin{array}{l}\text { Année de } \\
\text { publication }\end{array}$ & $\begin{array}{c}\text { Nombre de } \\
\text { colonnes }\end{array}$ & Pagination \\
\hline MERCATOR & L-M & 1904 & 10 & $1731-1736$ \\
\hline Mercatura & L-M & 1904 & 53 & $1743-1769$ \\
\hline NAVICULARIUS & N-Q & 1907 & 2 & 20-21 \\
\hline Negotiator & N-Q & 1907 & 7 & $41-45$ \\
\hline Negotium Gestio & $\mathrm{N}-\mathrm{Q}$ & 1907 & 6 & $47-50$ \\
\hline NeXum & N-Q & 1907 & 13 & $77-83$ \\
\hline Nomina Transcripticia & N-Q & 1907 & 2 & $97-98$ \\
\hline Obligatio & N-Q & 1907 & 14 & $133-140$ \\
\hline OBvugulatio & N-Q & 1907 & 1 & 141 \\
\hline SOlutio & $\mathrm{R}-\mathrm{S}$ & 1911 & 7 & $1392-1395$ \\
\hline
\end{tabular}

Tableau 3 - Paul Huvelin et le DAGR

Il est impossible de savoir comment lui fut adressée l'invitation à participer au $D A G R$ sans une analyse préalable de sa correspondance passive, ce qui reste à faire. L'initiative peut être partie de Saglio, en raison de ses contacts dans le domaine du droit. Elle peut aussi être venue de Pottier, bien placé au sein de l'Université française. Quoi qu'il en soit, il faut souligner le fait que les travaux d'Huvelin étaient bien connus dans le milieu des antiquisants. Ses articles et ses livres relatifs à l'histoire du droit et du commerce pendant l'Antiquité sont cités assez fréquemment dans les revues spécialisées $^{27}$. Il écrivit lui-même pour le Bulletin de correspondance hellénique des commentaires sur un décret commercial grec qui venait d'être découvert alors ${ }^{28}$.

26 Durkheim fit mention de sa lecture d'Huvelin dans les lettres adressées à Mauss (É. Durkheim, Lettres à Marcel Mauss, Paris, 1998, p. 123). Voir aussi le compte rendu écrit par Durkheim à propos d'un des livres d'Huvelin, É. DurkheIM, "Tablettes magiques et le droit romain ", L'Année sociologique 6 (1903), p. 388-390.

27 Tout en excluant les innombrables allusions faites aux travaux d'Huvelin par certains collaborateurs de L'Année sociologique, voir dans ce sens : P. PERDRIZET, "Isopséphie ", Revue des Études Grecques (1904), p. 350-360 ; C. RuelLe, "Bibliographie annuelle des études grecques ", Revue des Études Grecques (1909), p. 444 ; H. LECHAT, "Une guerre d'usure : la deuxième guerre punique (compte rendu) ", Revue des Études Anciennes (1917), p. 188-189 ; et W. Deonna « Le portrait de Phidias sur le Bouclier de l'Athèna Parthénos ", Revue des Études Anciennes (1920), p. 291-308.

28 P. Huvelin, E. Schulhof, "Fouilles de Délos (1905) : Inscriptions ; loi réglant la vente du bois et du charbon à Délos ", Bulletin de correspondance hellénique (1907), p. 46-93. 
Quant aux notices publiées par Huvelin dans le $D A G R$, elles sont très révélatrices des domaines propres à sa spécialité, ce qui montre combien son recrutement fut conscient. D'un côté, nous trouvons des textes portant sur l'analyse de l'histoire des pratiques commerciales et des législations qui leur étaient sous-jacentes. Tel est le cas des notices mercator, mercatura, navicularius, negociator, negotium gestio et nomina transscripticia. D'un autre côté, des textes lui furent commandés sur des aspects précis $\mathrm{du}$ droit romain, pas nécessairement en rapport avec le commerce, comme nexum, obligatio, obvugulatio et solutio. Il est inutile de réaliser ici une description complète de chaque cas, tant est grande la complexité des contextes historiques analysés. À l'inverse, nous présenterons dans les grandes lignes ce qui confere à l'ensemble son unité.

Mises à part les très brèves notices techniques comme navicularius, nomina transcripticia et obvugulatio, la collaboration d'Huvelin attire l'attention par la combinaison qu'elle opère entre un récit historique et un comparativisme sociologiquement informé. Ainsi, par exemple, il se prévaut d'un phénomène comme l'afflux de l'or et de l'argent à partir de la conquête de l'Amérique pour expliquer au lecteur l'impact commercial de l'expansion macédonienne (DAGR, 1904, p. 1754-1756). Il met aussi en parallèle le transport pratiqué dans l'Europe moderne avec celui du monde méditerranéen ancien (DAGR, 1907, p. 42-45). Enfin, il évoque des faits connus du droit germanique pour éclairer certaines pratiques romaines (DAGR, 1907, p. 79). Il ne s'agissait pourtant pas d'un comparatisme uniforme. En fait, la comparaison était dirigée pour appréhender les spécificités de plans de la réalité distincts. Surtout dans le cas des notices touchant l'histoire du droit commercial, Huvelin propose aussi bien une histoire externe du commerce, confirmant les pratiques qui le configuraient dans les sociétés anciennes et leurs changements survenus au fil des siècles, qu'une histoire interne, en l'occurrence l'analyse des lois dans leurs rapports avec les transformations sociales et avec la tradition juridique elle-même.

Une autre donnée importante à souligner est le lien existant entre ses notices et les grands programmes de recherche des fondateurs de L'Année sociologique. Tout comme ces derniers, mais sans les citer, Huvelin parle de division du travail, de solidarité, de groupes familiaux, de magie et de religion. Néanmoins, cette adhésion ne va pas sans réserve. Effectivement, en étudiant l'autonomisation de la législation comme un indice de la complexification des sociétés antiques, Huvelin ne l'associe pas, comme l'eût fait Durkheim, à la solidarité organique ${ }^{29}$. L'affaiblissement du droit pénal fondé sur les lois du sang et imprégné par un fond magico-religieux n’était pas nécessairement le reflet d'une augmentation de cette solidarité ; il pouvait tout autant être l'indice d'une sophistication des mécanismes visant à produire des inégalités sociales nuisibles à son implantation.

Il est très malaisé d'évaluer l'impact de ces textes spécifiques d'Huvelin parmi les latinistes, les hellénistes et les sociologues. Bien que ses travaux aient connu une circu-

29 Voir spécialement : DAGR, 1904, p. 1744-1746;1907, p. 79-83 et p. 133-135. 
lation plutôt bonne dans ces cercles, nous n'avons trouvé que deux références à la participation d'Huvelin au DAGR. La première, chronologiquement, a été faite par Georges Seure. Dans le Bulletin de correspondance hellénique publié en 1912, il cite l'article negotiator ${ }^{30}$. L'autre référence, cette fois-ici à l'article obligatio, est venue d'un jeune helléniste qui, à l'époque, s'était également engagé dans la sociologie : Louis Gernet. Dans un texte sur le droit primitif en Grèce publié dans la Revue des Études Grecques en 1917, il utilise des hypothèses d'Huvelin ${ }^{31}$. Il est toutefois possible que ses contemporains aient tout simplement préféré ne se référer qu'aux travaux plus conséquents de l'auteur. Ce dernier y renvoie effectivement dans ses copieuses notes accompagnant les textes du $D A G R$, spécialement à ses articles, notamment celui qu'il publia dans L'Année sociologique, et aussi à sa thèse.

\section{Conclusion : note sur la place de la sociologie dans le $D A G R$ et sur la place du DAGR dans la sociologie}

Considérant les cinq volumes du DAGR publiés après la sortie de L'Année sociologique, nous constatons que la présence de la sociologie durkheimienne fut modeste en comparaison des dimensions de l'ouvrage (sur les 4819 pages publiées entre 1900 et 1919, 86 seulement ont été rédigées par des sociologues). En fait, il pouvait difficilement en aller autrement : l'importance donnée à la vie matérielle et au quotidien des Anciens laissait un espace secondaire aux grands essais d'interprétation socio-historique, où la sociologie durkheimienne pouvait jouer un rôle important. Petit ne veut toutefois pas dire inexistant. Ce fut surtout dans les articles liés aux aspects plus généraux des croyances et des pratiques religieuses que, outre les textes d'Henri Hubert et de Paul Huvelin, la sociologie durkheimienne se rendit présente. Jules Toutain, dans sacrificium et religio, cite les travaux collectifs de Mauss et d'Hubert, de même que la traduction française du manuel de Chantepie de la Saussaye, réalisée sous la direction d'Hubert ${ }^{32}$. De la même façon, Gustave Fougères, dans sa notice maimaktéria, fait allusion à l'Essai sur le Sacrifice (DAGR, 1904, p. 1555). Le fait est que les sociologues durkheimiens étaient devenus incontournables, du moins quant aux théories sur la pratique religieuse. Que ce soit pour marquer son accord ou pour signifier son désaccord par rapport à eux, il fallait nécessairement les citer. Quant aux autres champs de la sociologie, économique et juridique, et pour évoquer ici les divisions qui existaient au sein de L'Année sociologi$q u e$, il fallut attendre un peu plus longtemps. L'exception dans ce cas fut Huvelin, mais son autorité n'était reconnue que dans des domaines très spécifiques.

30 G. Seure, "Antiquités thraces de la Propontide ", Bulletin de correspondance hellénique (1912), p. 557 et 614.

31 L. GeRnET, "Hypothèses sur le contrat primitif en Grèce ", Revue des Études Grecques (1917), p. 249-293, 363-383.

32 DAGR, 1911, p. 344 et 454. 
L'autre facette de notre investigation est de considérer le DAGR sous l'angle de l'institutionnalisation de la sociologie. Connaissant l'espace prédominant des études gréco-latines dans l'ensemble du système d'enseignement, et en particulier au sein des facultés de lettres, les mêmes qui virent naitre les premières chaires de sociologie, nous pouvons dire certainement que cette perspective ne manque pas d'intérêt. Dans la mesure où certains chercheurs proches de Durkheim se firent représenter dans le $D A G R$, soit dans des textes directs, soit dans des citations, cela avait des répercussions sur les investissements des antiquisants. De plus, parce qu'elle venait à figurer dans le hall d'entrée des savoirs légitimement applicables à l'étude du monde antique, la sociologie pouvait aussi donner de la valeur aux investissements intellectuels des générations nouvelles. L'augmentation du nombre d'hellénistes dans les filières de L'Année sociologique jusqu'à la publication du dernier numéro de sa première série, en 1913, ne nous semble pas fortuite. Louis Gernet, Henri Jeanmaire et Pierre Roussel, tandis qu'ils suivaient la voie de la spécialisation, parièrent sur la sociologie comme sur quelque chose qui pouvait renforcer leur projet de carrière. Parmi les latinistes, un raisonnement similaire peut s'appliquer à André Piganiol, même s'il n’a pas écrit pour la première série. Par conséquent, il nous paraît que les articles d'Hubert et d'Huvelin dans le DAGR, plus qu'ils n'ont exercé un impact sur les trajectoires individuelles de tel ou tel individu, ont permis d'élargir les espaces d'insertion institutionnelle d'un groupe.

Rafael Faraco BeNTHIEN

Université de São Paulo (doctorant) et Boursier FAPESP

Rua Dr. Nogueira Martins, 420, ap. 83. Saúde.

04143-020 São Paulo - SP - Brésil.

rfbenthien@hotmail.com 